\title{
Multiobjective CVaR Optimization Model and Solving Method for Hydrothermal System Considering Uncertain Load Demand
}

\author{
Zhongfu Tan, Liwei Ju, Huanhuan Li, Chao Qin, and Daoxin Peng \\ School of Economics and Management, North China Electric Power University, Beijing 102206, China \\ Correspondence should be addressed to Liwei Ju; 183758841@qq.com
}

Received 30 December 2014; Accepted 20 May 2015

Academic Editor: Eric Florentin

Copyright (C) 2015 Zhongfu Tan et al. This is an open access article distributed under the Creative Commons Attribution License, which permits unrestricted use, distribution, and reproduction in any medium, provided the original work is properly cited.

\begin{abstract}
In order to solve the influence of load uncertainty on hydrothermal power system operation and achieve the optimal objectives of system power generation consumption, pollutant emissions, and first-stage hydropower station storage capacity, this paper introduced CVaR method and built a multiobjective optimization model and its solving method. In the optimization model, load demand's actual values and deviation values are regarded as random variables, scheduling objective is redefined to meet confidence level requirement and system operation constraints and loss function constraints are taken into consideration. To solve the proposed model, this paper linearized nonlinear constraints, applied fuzzy satisfaction, fuzzy entropy, and weighted multiobjective function theories to build a fuzzy entropy multiobjective CVaR model. The model is a mixed integer linear programming problem. Then, six thermal power plants and three cascade hydropower stations are taken as the hydrothermal system for numerical simulation. The results verified that multiobjective CVaR method is applicable to solve hydrothermal scheduling problems. It can better reflect risk level of the scheduling result. The fuzzy entropy satisfaction degree solving algorithm can simplify solving difficulty and get the optimum operation scheduling scheme.
\end{abstract}

\section{Introductions}

Electric power system scheduling means when load demand is met, units' power generation assignment is allocated according to minimized energy consumption or power generation cost goal, which takes system operation output, power climbing, backup service, and other constraints into consideration $[1,2]$. Thermal power and hydro power are the primary power types in China. The hydrothermal power joint scheduling can achieve energy-saving requirements and reduce system power generation's average cost [3]. So the study on hydrothermal power joint scheduling has important theoretical value and practical significance.

Currently, many researchers have already studied on hydrothermal power joint scheduling optimization problems. Existing literatures mainly focus on 3 aspects, namely, inflow forecast, scheduling model, and solving method building. On aspect of inflow forecast, it generally uses load demand and inflow historical data and classical forecast methods $[4,5]$, modern forecast methods [6-8], and intelligence forecast methods [9-11] to forecast load demand and inflow in the next period of time. However, during practical applications the methods above can get quite accurate forecast results but their forecast accuracy can hardly meet system scheduling requirements.

As for hydrothermal power joint scheduling optimization model, there are two research priorities, namely, uncertain problems and environmental requirements. Because of the uncertainty of inflow and load demand, units' output should be allocated according to load forecast results. So the core of the scheduling model is prior-decision. Generally, fuzzy decision theory [12], random optimization theory [13], opportunity theory [14], and other theories are introduced to build optimization model and solve prior-decision problems. Influenced by environmental pressure, minimized pollutant emission has become another optimize point [15], which makes system scheduling optimization problems transforming from single objective to multiobjective.

The introduction of uncertain factors and new scheduling objectives makes scheduling models becoming more complex. Multivariable, multiconstraint, multiobjective, nonconvexity, and nonlinear problems need corresponding 
algorithms to get ideal scheduling result. In existing literatures there are conventional solving algorithms and intelligent solving methods, such as calculus of variations, dynamic planning, differential evolution algorithm, genetic algorithms, and artificial neural network algorithm [16-20]. However, conventional algorithms usually have problems in defining parameters, transferring constraints that they could hardly achieve high optimization degree [21]. Intelligent algorithms can overcome those shortcomings. But when individual's extreme values do not meet multiobjective planning principles, intelligent algorithms are easy to fall into local extreme point and the algorithm's searching capability is limited [22].

According to the above overview, existing researches about hydrothermal joint scheduling are mainly concentrated in model building when uncertain factors are considered, and few on risk analysis, prevention, and control about what the uncertain factors would bring to the system. In China, hydropower resources are mainly concentrated in southern regions. Reservoir storage capacity is quite big and inflow uncertainty is small [23]. Therefore this paper does not take inflow uncertainty into consideration and only considers load demand uncertainty.

The rest of the paper is organized as follows. In Section 2, the basic mathematic model is construct which take the minimum power generation cost, the minimum pollutant emission, and the maximum storage capacity of the hydropower station as the optimization objectives. Section 3 introduces the multiobjective CVaR method and built a hydrothermal joint scheduling optimization model. In order to solve the proposed model in Section 3, Section 4 linearizes nonlinear constraints, used fuzzy satisfaction degree theory to blur the objective function, and used entropy theory to weight the multiobjective function. The model was transferred into a fuzzy entropy multiobjective CVaR model. In Section 5, six thermal plants and three cascade hydropower stations are taken as the simulation system to verify the effectiveness and feasibility of the proposed model and solving method. Section 6 highlights the main conclusions of the paper.

\section{Basic Mathematic Model}

2.1. Objective Functions. Currently, the objectives functions of the hydrothermal system scheduling optimization model consist of minimized power generation cost, minimized pollutant emission, and maximized storage capacity of the hydropower station.

(1) Minimized power generation energy consumption objective function is as follows:

$$
\begin{aligned}
& \min f_{1}=\sum_{t=1}^{T} \sum_{i=1}^{I}\left(u_{i, t} f_{c}\left(g_{i, t}\right)+u_{i, t}\left(1-u_{i, t-1}\right) D_{i, t}\right), \\
& f_{c}\left(g_{i, t}\right)=a_{i}+b_{i} g_{i, t}+c_{i} g_{i, t}^{2},
\end{aligned}
$$

wherein $f_{1}$ is system's power generation energy consumption cost; $I$ is the number of thermal units; $T$ is the cycle of power generation scheduling, generally 24 hours; $u_{i, t}$ is an $0-1$ variable; and $u_{i, t}=1$ means the unit $i$ is in operating; otherwise it is not in operating. $g_{i, t}$ is the output of unit $i$ at time $t ; a_{i}, b_{i}$, and $c_{i}$ are fuel cost parameters of unit $i$, which can be calculated according to historical data of the unit. $D_{i, t}$ is the startup-shutdown cost of thermal unit $i$ at time $t$ :

$$
\begin{aligned}
D_{i, t} & = \begin{cases}D_{i}^{h}, & T_{d, i}^{\mathrm{min}}<T_{i}^{\mathrm{off}}(t) \leq H_{i}^{\mathrm{off}} \\
D_{i}^{c}, & T_{i}^{\mathrm{off}}(t)>H_{i}^{\mathrm{off}},\end{cases} \\
H_{i}^{\mathrm{off}} & =T_{d, i}^{\mathrm{min}}+T_{s, i}^{c},
\end{aligned}
$$

wherein $D_{i}^{c}$ is the cold-start cost of unit $i$; $D_{i}^{h}$ is the hotstart cost of unit $i$; $T_{d, i}^{\mathrm{min}}$ is the minimum allowed continuous downtime of unit $i$; $T_{i}^{\text {off }}(t)$ is the continuous downtime of unit $i$ at time $t ; T_{s, i}^{c}$ is the cold-start time of unit $i ; H_{i}^{\text {off }}$ is the summation of minimum downtime and cold-start time of unit $i$.

(2) Minimized pollutant emission objective function is as follows:

$$
\min f_{2}=\sum_{t=1}^{T} \sum_{i=1}^{I} \sum_{m=1}^{M}\left\{u_{i, t}\left[\omega_{i}^{m}+\beta_{i}^{m} g_{i, t}+\gamma_{i}^{m} g_{i, t}^{2}\right]\right\},
$$

wherein $M$ is the number of pollutant types and $\omega_{i}^{m}, \beta_{i}^{m}$, and $\gamma_{i}^{m}$ are pollutant emission parameters of unit $i$.

(3) Maximized storage capacity of the first-level hydropower station is as follows:

$$
\begin{aligned}
\max f_{3}= & \sum_{t=1}^{T} \sum_{j=1}^{J} V_{j t}, \\
V_{j t}= & V_{j, t-1}+\widehat{Q}_{t}-Q_{t}-S_{j t} \\
& +\sum_{h=1}^{H}\left(Q_{h, t-\tau_{h j}}+S_{h, t-\tau_{h j}}\right),
\end{aligned}
$$

wherein $V_{j t}$ is the water storage capacity od hydropower station $j$ at time $t ; \widehat{Q}_{t}$ is the inflow volume of the superiors hydropower station at time $t ; Q_{t}$ is the discharged flow of hydropower station $j$ at time $t ; S_{j t}$ is the nature abandoned water of hydropower station $j$ at time $t ; H$ is the number of the upstream hydropower units that directly connected with hydro unit $j ; \tau$ is the flow delay between hydropower unit $j$ and upstream hydropower unit $h$.

2.2. Constraints. (1) Load demand and supply balance constraint is as follows:

$$
\sum_{i=1}^{I} g_{i, t} u_{i, t}+\sum_{j=1}^{J} g_{j, t}-g_{t}^{l}=L(t),
$$

wherein $L(t)$ is the load demand at time $t ; g_{j, t}$ is the output of hydropower station $j$ at time $t ; g_{t}^{l}$ is system's total power loss at time $t$.

(2) Thermal units' output constraint is as follows.

Thermal units' output constraints include upper and lower power generation limit constraint, power climbing 
limit constraint, the shortest start time constraint, and the shortest downtime constraint. The detailed constraint functions are (7)-(9). Consider the following:

$$
u_{i t} g_{i}^{\min } \leq g_{i t} \leq u_{i t} g_{i}^{\max },
$$

wherein $g_{i}^{\max }$ and $g_{i}^{\min }$ are the power generation output upper and lower limit of unit $i$ :

$$
\Delta g_{i}^{-} \leq g_{i t}-g_{i, t-1} \leq \Delta g_{i}^{+},
$$

wherein $\Delta g_{c i}^{-}$and $\Delta g_{c i}^{+}$are power climbing limits:

$$
\begin{aligned}
& \left(T_{i, t-1}^{\text {on }}-M_{i}^{\text {on }}\right)\left(u_{i, t-1}-u_{i t}\right) \geq 0, \\
& \left(T_{i, t-1}^{\text {off }}-M_{i}^{\text {off }}\right)\left(u_{i t}-u_{i, t-1}\right) \geq 0,
\end{aligned}
$$

wherein $M_{i}^{\text {on }}$ is the shortest startup time of thermal unit $i$; $T_{i, t-1}^{\mathrm{on}}$ is the continuous operation time of unit $i$ at time $t-1$; $M_{i}^{\text {off }}$ is the shortest downtime of unit $i ; T_{i, t-1}^{\text {off }}$ is the continuous downtime of unit $i$ at time $t-1$.

(3) Hydropower units' output constraints are as follows.

Hydropower units' output constraints mainly include units' power generation flow constraint, storage capacity restrictions constraint, and dynamic water balance constraint. The detailed constraint functions are (10)-(12). Consider the following:

$$
\begin{aligned}
g_{j, t}= & c_{0 j}+c_{1 j} Q_{j t}+c_{2 j} V_{j t}+c_{3 j} Q_{j t} V_{j t}+c_{4 j} Q_{j t}^{2} \\
& +c_{5 j} V_{j t}^{2},
\end{aligned}
$$

wherein $Q_{j t}$ is the flow of hydropower unit $j$ at time $t ; V_{j t}$ is the storage capacity of hydropower unit $j$ at time $t ; c_{0 j}, c_{1 j}$, $c_{2 j}, c_{3 j}, c_{4 j}$, and $c_{5 j}$ are output parameters of hydropower unit $j$, which can be calculated according to the historical data of the unit:

$$
\begin{aligned}
& Q_{j}^{\min } \leq Q_{j t} \leq Q_{j}^{\max }, \\
& V_{j}^{\min } \leq V_{j t} \leq V_{j}^{\max },
\end{aligned}
$$

wherein $Q_{j}^{\min }$ and $Q_{j}^{\max }$ are, respectively, the minimum and maximum power generation flow of hydropower unit $j$; $V_{j}^{\min }$ and $V_{j}^{\max }$ are, respectively, the lower and upper storage capacity limit of hydropower unit $j$ :

$$
\begin{aligned}
& V_{j T} \\
& \quad=V_{j 0} \\
& \quad+\sum_{t=1}^{T}\left[\widehat{Q}_{t}-Q_{t}-S_{j t}+\sum_{h=1}^{H}\left(Q_{h, t-\tau_{h j}}+S_{h, t-\tau_{h j}}\right)\right], \\
& \left.V_{j t}\right|_{t=0}=V_{j 0}, \\
& \left.V_{j t}\right|_{t=T}=V_{j T},
\end{aligned}
$$

wherein $V_{j 0}$ and $V_{j T}$ are, respectively, storage capacities at the initial time and end time of hydropower unit $j$.

\section{Multiobjective CVaR Hydrothermal Joint Scheduling Optimization Model}

3.1. Description of Load Demand Uncertainty. When making hydrothermal power generation scheduling decision, the decision maker should allocate power generation assignment according to the forecast value of load demand. The forecast value is calculated based on historical data. And if load demand uncertainty is considered, the load demand and supply balance can be described as

$$
\sum_{i=1}^{I} g_{i, t} u_{i, t}+\sum_{j=1}^{J} g_{j, t}-g_{t}^{l} \geq L(t)
$$

wherein $\widetilde{L}(t)$ is the forecasted value of load demand.

The relationship between the actual value and forecasted value is

$$
L(t)=\widetilde{L}(t)+e(t)
$$

wherein $e(t)$ is the deviation value between the actual value and forecasted value, which, according to literature [24], obeys normal distribution $e(t) \sim N\left(\theta_{t}, \sigma_{t}^{2}\right) . \theta_{t}$ and $\sigma_{t}$ are, respectively, the average value and standard deviation of $e(t)$. Distribution law of $e(t)$ can be calculated according to historical data.

When the load demand uncertainty exists, there are two possible scenarios. When load demand forecasted value is higher than the actual value, some units may be forced to shut down. And when the forecasted value is lower than the actual value, the hydropower may not have full access to the grid, which would bring scheduling loss to the system. Load demand uncertainty can directly influence system's stability and bring corresponding risks. Therefore its necessary to use corresponding risk analysis method to study on the optimized decision method when uncertain factors are took into consideration.

3.2. Multiobjective CVaR Method. VaR method has a shortcoming that it can be applied to measure risk situation only when the confidence level is determined. CVaR method can overcome the shortcoming of $\mathrm{VaR}$ and describe risk distribution beyond the confidence. However, in practical CVaR method can only solve combinatorial optimization problems with single loss function. When system has multiple loss functions, CVaR need be expanded to multiobjective CVaR method [25]. Assume there is a loss function $f_{k}(x, \xi) \in$ $R^{n} \times R^{m} \rightarrow R^{1}(k=1,2, \ldots, K)$ that is related with decision variable $x \in \mathbf{X} \subset R^{n}$. The loss function is a continuous function. $\xi$ is a continuous random variable and its probability density function is $p(z)$. Then the distribution function of $f_{k}(x, \xi)$ is

$$
\psi_{k}\left(x, y_{k}\right)=P\left\{f_{k}(x, \xi) \leq y_{k}\right\} \int_{f_{k}(x, z) \leq y_{k}} p(z) d z
$$


Define $\lambda_{i}(i=1,2, \ldots, n)$ as weights of each loss function and $\alpha$-VaR loss value of decision variable $x$ under confidence level $\alpha$ and weight $\lambda$ is

$$
\begin{gathered}
y^{*}(x, \lambda)=\min \left\{y \mid \sum_{i=1}^{K} \lambda_{k}\left(1-\alpha_{k}\right)^{-1} \psi_{k}(x, \xi)\right. \\
\left.\geq \sum_{k=1}^{K} \lambda_{k} \alpha_{k}\left(1-\alpha_{k}\right)^{-1}\right\} .
\end{gathered}
$$

Introduce loss function $\phi_{k, \alpha_{k}}\left(x, y_{k}\right)$ :

$$
\phi_{k, \alpha_{k}}\left(x, y_{k}\right)=\left(1-\alpha_{k}\right)^{-1} \int_{f_{k}(x, z) \geq y_{k}} f(x, z) p(z) d z .
$$

Then the optimum $\alpha$-CVaR loss value $\phi\left(x, y^{*}(x, \lambda)\right)$ of decision variable $x$ under confidence level $\alpha$ and weight $\lambda$ is

$$
\phi\left(x, y^{*}(x, \lambda)\right)=\sum_{i=1}^{n} \lambda_{i} \phi_{i, \alpha_{i}}\left(x, y^{*}(x, \lambda)\right),
$$

wherein $\alpha_{i}$ is the confidence levels of loss function $i$.

To find the minimum $\alpha$-CVaR loss value, $\phi\left(x, y^{*}(x, \lambda)\right)$ should achieve the minimum value $x$ on $X$. And the optimization problem can be defined as

$$
\begin{aligned}
(\mathrm{P} 1) \min \quad \phi & \left(x, y^{*}(x, \lambda)\right) \\
& =\sum_{k=1}^{K} \lambda_{k} \phi_{k, \alpha_{k}}\left(x, y^{*}(x, \lambda)\right)
\end{aligned}
$$

$$
\text { s.t. } x \in X \text {. }
$$

To simplify the solving progress of model (P1), this paper redefined loss functions and their optimization problems. The detailed model is model (P2):

$$
\begin{aligned}
F_{k, \alpha_{k}}\left(x, y_{k}\right)= & y_{k}+\left(1-\alpha_{k}\right)^{-1} \int_{z \in R^{m}}\left(f_{k}(x, z)-y_{k}\right)^{+} p(z) d z, \\
\text { (P2) } \min & \sum_{k=1}^{K} \lambda_{k} F_{k, \alpha_{k}}(x, y) \\
\text { s.t. } & y \in R, x \in X .
\end{aligned}
$$

The equivalent problem of model (P2) is as follows: for any $x$, if $\min \sum_{k=1}^{K} \lambda_{k} F_{k, \alpha_{k}}(x, y)$ has a unique and optimal solution $y . y$ meets the following constraint:

$$
P\left(\pi_{k}(x, y)=\xi\right)=\int_{\pi_{k}(x, z)=\xi} p(z) d z=0 .
$$

Then, the following conclusion can be gotten:

$$
\begin{aligned}
\sum_{i=1}^{n} \lambda_{k} \phi_{k, a_{k}}(x, y) & =\min _{\xi \in R} \sum_{k=1}^{K} \lambda_{k} F_{k, \alpha_{k}}(x, y), \\
y & =y^{*}(x, \lambda) .
\end{aligned}
$$

For any determined weight value $\lambda$, if $(x, y)$ could make model (P2) achieve the optimum and (2) is tenable, then $x$ could make model (P1) achieve the optimum.
3.3. Multiobjective CVaR Scheduling Model. When load demand uncertainty is considered, load deviation would be undertaken by thermal units and hydro units. Transfer system scheduling objective functions referring to the literature [24] and get the new objective function, which take load demand uncertainty into consideration:

$$
\begin{aligned}
& \min f_{1}=\sum_{t=1}^{T} \sum_{i=1}^{I}\left(u_{i, t} f_{c}\left(g_{i, t}+\varphi_{i} e_{t}\right)+u_{i, t}\left(1-u_{i, t-1}\right) D_{i, t}\right), \\
& \min f_{2} \\
& =\sum_{t=1}^{T} \sum_{i=1}^{I} \sum_{m=1}^{M}\left\{u_{i, t}\left[\omega_{i}^{m}+\beta_{i}^{m}\left(g_{i, t}+\varphi_{i} e_{t}\right)+\gamma_{i}^{m}\left(g_{i, t}+\varphi_{i} e_{t}\right)^{2}\right]\right\}, \\
& \max f_{3}=\sum_{t=1}^{T} \sum_{j=1}^{J} V_{j t}^{\prime}, \\
& V_{j t}^{\prime}=V_{j, t-1}+\widehat{Q}_{t}-Q_{t}-S_{j t}-\frac{\varphi_{j} e_{t}}{\rho_{j}}+\sum_{h=1}^{H}\left(Q_{h, t-\tau_{h j}}+S_{h, t-\tau_{h j}}\right),
\end{aligned}
$$

wherein $\varphi_{i}$ and $\varphi_{j}$ are, respectively, task proportions of thermal units and hydro units and $\rho_{j}$ is the output power coefficient of hydro units.

After considering uncertainties, traditional constraints are needed to converse to uncertainty constraints, as follows:

$$
\begin{aligned}
\varphi_{i} e_{t}+\Delta g_{i}^{-} \leq & g_{i t}-g_{i, t-1} \leq \Delta g_{i}^{+}-\varphi_{i} e_{t}, \\
g_{j, t}+\varphi_{j} e_{t}= & c_{0 j}+c_{1 j} Q_{j t}+c_{2 j} V_{j t}^{\prime}+c_{3 j} Q_{j t} V_{j t}^{\prime}+c_{4 j} Q_{j t}^{2} \\
& +c_{5 j} V_{j t}^{\prime 2} .
\end{aligned}
$$

Further, multiobjective CVaR approach is used to build the hydrothermal optimization scheduling model. First, define a schedule loss function, as follows:

$$
\begin{aligned}
& F_{k, \alpha_{k}}\left(G, y_{k}\right) \\
& =y_{k}+\left(1-\alpha_{k}\right)^{-1} \int_{z \in R^{m}}\left(f_{k}(G, e)-y_{k}\right)^{+} p(e) d e, \\
& k=1,2,3,
\end{aligned}
$$

wherein $G$ is units output set of the system; $\alpha_{k}$ is the confidence level requirement of objective function $f_{k} ; y_{k}$ is decision maker's expected value of objective function $f_{k}$.

Then build a hydrothermal joint scheduling optimization model which sets minimized $\alpha$-CVaR loss value as its objective:

$$
\begin{array}{ll}
\min & F_{k, \alpha_{k}}\left(G, y_{k}\right), \quad k=1,2 \\
\max & F_{3, \alpha_{3}}\left(G, y_{3}\right) \\
\text { s.t. } & \sum_{i=1}^{I} g_{i, t} u_{i, t}+\sum_{j=1}^{J} g_{j, t}-g_{t}^{l} \geq \widetilde{L}(t)+e(t) \\
& (1)-(14),(24),(25) \\
& G=\left\{g_{i t}, g_{j t}\right\} \\
& i=1,2, \ldots, n ; j=1,2, \ldots, J .
\end{array}
$$




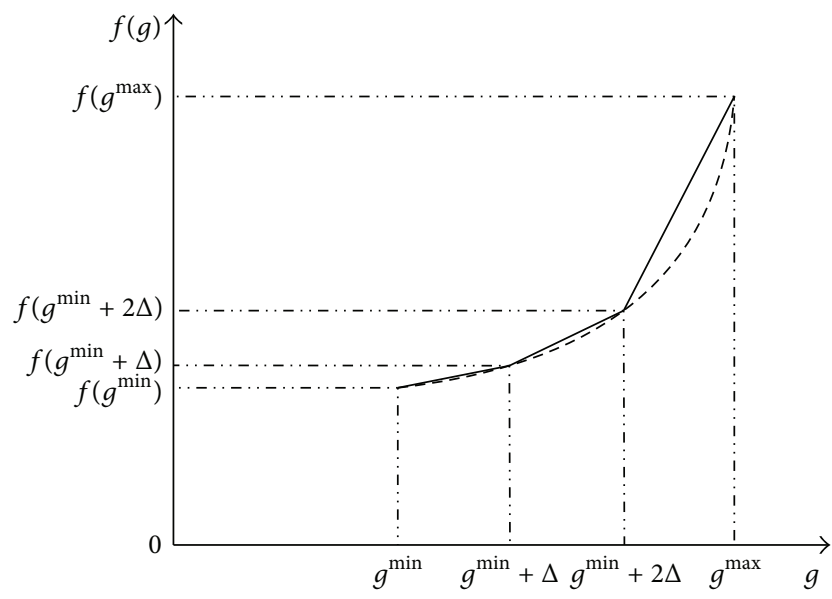

FIGURE 1: Linear processing of quadratic function.

\section{Entropy Fuzzy Satisfaction Degree Solving Method}

According to (25), solve hydrothermal joint scheduling optimization model that needs two key elements, namely, objective functions' weights and the optimum acceptable degree $y_{k}$ of each objective. This section puts forward entropy fuzzy satisfaction degree solving model. Entropy theory is used to get weight for each objective, and fuzzy satisfaction degree is used to simplify objective functions and get the optimum $y_{k}$.

4.1. Linearization Processing. According to (1) and (2), when an objective function is a quadratic function, the objective function should be simplified linearization processing. Divide thermal units' power limits $\left[g_{i}^{\min }, g_{i}^{\max }\right]$ into $N$ segments. Then function can be expressed as a piecewise function. When $g_{i, t} \in\left[g_{i}^{\min }+n \Delta, g_{i}^{\min }+(n+1) \Delta\right]$,

$$
\begin{aligned}
& f_{c}^{\prime}\left(g_{i t}\right) \\
& =f_{c}^{\prime}\left(g_{i}^{\min }+n \Delta\right) \\
& \quad+\left(g_{i t}-g_{i}^{\min }-n \Delta\right)\left[b_{i}+(2 n+1) c_{i} \Delta+2 c_{i} g_{i}^{\min }\right],
\end{aligned}
$$

wherein $n=0,1, \ldots, N-1, \Delta=\left(g_{i}^{\max }-g_{i}^{\min }\right) / N$. According to the literature [26], when $N \geq 5$, the maximum relative error of objective function should be less than $1 \%$, generally less than $0.5 \%$, and in segmented approaching situation the error is approaching to 0 . Figure 1 shows the linearization progress of a quadratic function.

4.2. Blurring Processing. The optimization direction of the first level cascade hydropower station objective function is maximized, while the other two are minimized. Therefore this section introduces declining semigradient membership degree function and rising semilinear membership degree function to blur the objectives. The detailed progress is as follows.
TABLE 1: The input-output tables of objective function.

\begin{tabular}{llll}
\hline Objective function & $F_{1}$ & $F_{2}$ & $F_{3}$ \\
\hline$F_{1}{ }^{*}$ & $F_{1}{ }^{\min }$ & $F_{1}{ }^{(2)}$ & $F_{1}{ }^{(3)}$ \\
\hline$F_{2}{ }^{*}$ & $F_{2}{ }^{(1)}$ & $F_{2}{ }^{\min }$ & $F_{2}{ }^{(3)}$ \\
\hline$F_{3}{ }^{*}$ & $F_{3}{ }^{(1)}$ & $F_{3}{ }^{(2)}$ & $F_{3}{ }^{\max }$ \\
\hline
\end{tabular}

Note: objective with $*$ means the objective is the optimization objective.

(1) Input the initial data and solve each single optimization objective, respectively, to get functions' value of different optimization objectives. Form a decision properties table of the objective functions, as shown in Table 1.

(2) Scale each objective according to decision makers' wishes and preferences, and define $F_{1}^{\max }, F_{2}^{\max }$, and $F_{3}^{\min }$ and their value range. The equations are

$$
\begin{aligned}
0 & \leq F_{1}^{\mathrm{max}}-F_{1}^{\mathrm{min}} \\
& \leq\left\{F_{1}^{(2)}-F_{1}^{\mathrm{min}}, F_{1}^{(3)}-F_{1}^{\mathrm{min}}\right\}, \\
0 & \leq F_{2}^{\mathrm{max}}-F_{2}^{\min } \\
& \leq\left\{F_{2}^{(1)}-F_{2}^{\min }, F_{2}^{(3)}-F_{2}^{\min }\right\}, \\
F_{3}^{\max }-F_{3}^{\min } & \geq\left\{F_{3}^{\max }-F_{3}^{(1)}, F_{3}^{\max }-F_{3}^{(2)}\right\} .
\end{aligned}
$$

(3) Use declining semigradient membership degree function to deal with minimized power generation cost objective function and minimized pollutant emission objective function, and use rising semilinear membership degree function to deal with maximized storage capacity objective function. Then membership degree function could be defined:

$$
\begin{aligned}
& \pi_{i}\left(f_{i}\right)= \begin{cases}0, & F_{i} \geq F_{i}^{\max } \\
\frac{F_{3}^{\max }-F_{3}}{F_{3}^{\max }-F^{\min }}, & F_{i}^{\min }<F_{i}<F_{i}^{\max } \\
1, & F_{i} \leq F_{i}^{\min },\end{cases} \\
& \pi_{3}\left(f_{3}\right)= \begin{cases}1, & F_{3} \geq F_{3}^{\max } \\
\frac{F_{3}-F_{3}^{\min }}{F_{3}^{\max }-F^{\min }}, & F_{3}^{\min }<F_{3}<F_{3}^{\max } \\
0, & F_{3} \leq F_{3}^{\min },\end{cases}
\end{aligned}
$$

wherein $f_{i}^{\min }$ and $f_{i}^{\max }$ are, respectively, the minimum and maximum value of the objective function $f_{i}(\cdot) . \pi\left(f_{i}\right)$ is the membership degree function of the objective function $f_{i}(\cdot)$.

4.3. Weight Allocation. Multiobjective CVaR optimization problems require rational weight allocation to transfer multiobjective optimization problem into single objective optimization problem. Weight allocation methods can be classified into two types, namely, subjective weighting methods and objective weighting methods. Compared with subjective weighting methods, objective weighting methods have an 
advantage of avoiding influences from subjective experiences by directly calculating weights according to objective information. Entropy method is an objective weighting method. Entropy method calculates evaluation indexes' entropy and weight according to evaluation objectives' actual data. Because of its strong objectivity and simple calculation progress, entropy method is wide and used in many comprehensive evaluation problems. Based on Table 1, this section uses entropy method to calculate weight of each objective. Detailed introduction of the entropy meth refers to the literature [26], and the following is the calculation progress.

(1) Calculate entropy $E_{k}$ of objective function $k$ :

$$
E_{k}=-\psi \sum_{i=1}^{n} r_{i j} \ln \left(r_{i j}\right)
$$

wherein $\psi=1 / \ln (n)$ is a constant related with the specimen, and it was defined to make $E_{k} \in[0,1] ; r_{i j}$ meets $0<r_{i j}<1$ and $\sum_{i=1}^{n} r_{i j}=1$, when $r_{i j}=0, r_{i j} \ln \left(r_{i j}\right)=0$.

(2) Information deviation degree of objective function is as follows:

$$
d_{k}=1-E_{k}
$$

(3) Calculate weights of the objectives:

$$
\lambda_{k}=\frac{d_{k}}{\sum_{k=1}^{K} d_{k}}=\frac{\left(1-E_{k}\right)}{\left(K-\sum_{k=1}^{K} E_{k}\right)} .
$$

4.4. Entropy Fuzzy Multiobjective CVaR Model. Assume the allocated weight and membership degree of each objective are $\pi_{k}\left(f_{k}\right)$ and $\lambda_{k}$. If $\pi^{*}$ is the satisfaction degree of $\pi_{k}\left(f_{k}\right)$,

$$
\pi^{*}=\min \left\{\pi_{k}\left(f_{k}\right), k=1,2,3\right\} .
$$

According to the maximum and minimum principles of fuzzy set theory, multiobjective CVaR model could be transferred:

$$
\begin{array}{ll}
\min & \sum_{k=1}^{3} \lambda_{k} \pi_{k}\left(f_{k}\right) \\
\text { s.t. } & \pi_{1}\left(f_{1}\right) \geq \pi^{*} \\
& \pi_{2}\left(f_{2}\right) \geq \pi^{*} \\
& \pi_{3}\left(f_{3}\right) \geq \pi^{*} \\
& 0 \leq \pi^{*} \leq 1 \\
& \sum_{i=1}^{I} g_{i, t} u_{i, t}+\sum_{j=1}^{J} g_{j, t}-g_{t}^{l} \geq \widetilde{L}(t)+e(t) \\
& (1)-(14),(24),(25) \\
& i=1,2, \ldots, n ; j=1,2, \ldots, m .
\end{array}
$$

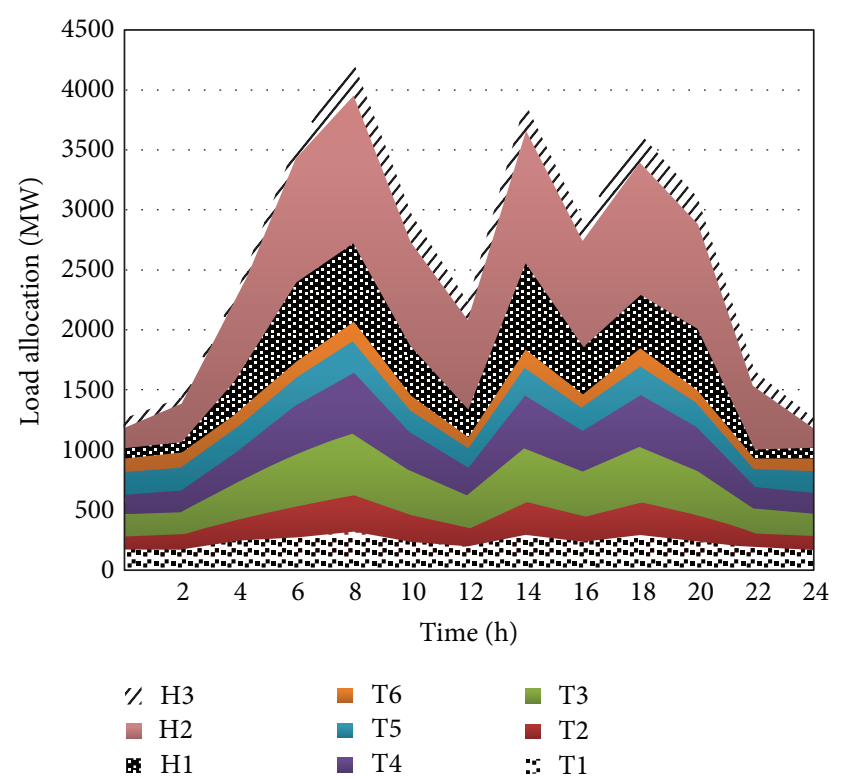

FIGURE 2: System unit output arrangement.

\section{Simulations and Analysis}

This section chooses 6 thermal plants (T1-T6) and 3 cascade hydropower stations (H1-H3) as simulation base. Thermal plants' basic parameters and comprehensive emission parameters refer to the literature [24]; cascade hydropower stations' operation parameters are listed in Table 2. Assume load forecast error value obeys normal distribution $e(t) \sim$ $\left(0,(30)^{2}\right)$, and both of T5 and T6 bear $20 \%$ of the forecast error. Choose a typical daily load distribution as load demand of the simulation, as listed in Table 3.

In the risk-neutral situation, meeting all minimized power generation cost, minimized pollutant emission, and maximized first-level hydropower station storage capacity objectives, system units' output assignment is drawn in Figure 2.

In risk nonneutral situations, to analyse the influence of risk attitude on units scheduling result, this section studies scheduling results under different confidence levels. This section, respectively, calculates scheduling results of each objective in two situations, namely, constant confidence level $\alpha$ and mutative confidence level $\alpha$. The detailed information is listed in Table 4.

First, optimize the objective function of each single objective scheduling scenario to get system units' output assignment and function value, and form objective function decision properties table. Each objective's boundary value can be calculated according to (24)-(26). And used entropy method can get objective functions' weights. Fuzzy objective membership degree function parameters and weights are listed in Table 5.

Compare different scenarios in Table 5 and find when all objective functions' confidence levels are the same, weights of the objective functions are basically the same, and the deviation is small. Otherwise when confidence levels are 
TABLE 2: Basic parameters of cascade hydropower stations.

\begin{tabular}{lcccc}
\hline ID & $\begin{array}{c}\text { Regulation } \\
\text { characteristics }\end{array}$ & Normal water level $/ \mathrm{m}$ & $\begin{array}{c}\text { Normal } \\
\text { capacity } /\left(10^{8} \mathrm{~m}^{3}\right)\end{array}$ & Dead water level/m \\
\hline H1 & Many years & 780 & 83.95 & 731 \\
H2 & $\begin{array}{l}\text { Run-off } \\
\text { H3 }\end{array}$ & 645 & 0.26 & 637 \\
\hline Run-off & Dead storage & 112 & 4.02 & 110 \\
\hline H1 & capacity $/\left(10^{8} \mathrm{~m}^{3}\right)$ & Initial capacity $/ \mathrm{m}$ & Installed & Hydropower output \\
capacity $/ \mathrm{MW}$ & $300 * 4$ & 0.9225 \\
Coefficient $/\left(\mathrm{MW} /\left(\mathrm{m}^{3} / \mathrm{s}\right)\right)$
\end{tabular}

TABLE 3: Demand load in typical daily.

\begin{tabular}{|c|c|c|c|c|c|c|}
\hline Time & 1 & 2 & 3 & 4 & 5 & 6 \\
\hline Load/MW & 1332 & 1254 & 1243 & 1431 & 1662 & 2343 \\
\hline Time & 7 & 8 & 9 & 10 & 11 & 12 \\
\hline Load/MW & 3630 & 3544 & 4162 & 4214 & 2998 & 2963 \\
\hline Time & 13 & 14 & 15 & 16 & 17 & 18 \\
\hline Load/MW & 2493 & 2218 & 3137 & 3873 & 3567 & 2896 \\
\hline Time & 19 & 20 & 21 & 22 & 23 & 24 \\
\hline Load/MW & 2896 & 3627 & 3901 & 3102 & 2260 & 1654 \\
\hline
\end{tabular}

TABLE 4: Simulation scenarios set.

\begin{tabular}{|c|c|c|c|c|c|c|}
\hline \multirow{2}{*}{ Objective function } & \multicolumn{3}{|c|}{ Same confidence level $\alpha$} & \multicolumn{3}{|c|}{ Different confidence levels $\alpha$} \\
\hline & Scenario 1 & Scenario 2 & Scenario 3 & Scenario 4 & Scenario 5 & Scenario 6 \\
\hline$\overline{f_{1}}$ & 0.85 & 0.9 & 0.95 & 0.85 & 0.9 & 0.95 \\
\hline$f_{2}$ & 0.85 & 0.9 & 0.95 & 0.9 & 0.85 & 0.9 \\
\hline$f_{3}$ & 0.85 & 0.9 & 0.95 & 0.95 & 0.95 & 0.85 \\
\hline
\end{tabular}

TABLE 5: Fuzzy target membership function parameters and weights.

\begin{tabular}{|c|c|c|c|}
\hline \multirow{2}{*}{ Scenario } & \multicolumn{3}{|c|}{ Fuzzy objective membership degree function parameters } \\
\hline & $F_{1} /$ ton & & $F_{1} /$ ton \\
\hline Scenario 1 & {$[5423,5648]$} & {$[6989,7532]$} & {$[3245,3573]$} \\
\hline Scenario 2 & {$[6372,6819]$} & {$[7989,8533]$} & {$[3645,4274]$} \\
\hline Scenario 3 & {$[7572,8820]$} & {$[9689,11234]$} & {$[4245,5075]$} \\
\hline Scenario 4 & {$[5223,5848]$} & {$[7589,8233]$} & {$[4135,4868]$} \\
\hline Scenario 5 & {$[6274,6652]$} & {$[6675,7143]$} & {$[4038,5260]$} \\
\hline Scenario 6 & {$[7342,8510]$} & {$[6649,7208]$} & {$[3428,4516]$} \\
\hline \multirow{2}{*}{ Scenario } & \multicolumn{3}{|c|}{ Fuzzy objective function weights } \\
\hline & $\lambda_{1}$ & $\lambda_{2}$ & $\lambda_{3}$ \\
\hline Scenario 1 & 0.324 & 0.333 & 0.343 \\
\hline Scenario 2 & 0.326 & 0.326 & 0.348 \\
\hline Scenario 3 & 0.332 & 0.329 & 0.339 \\
\hline Scenario 4 & 0.305 & 0.313 & 0.382 \\
\hline Scenario 5 & 0.332 & 0.303 & 0.365 \\
\hline Scenario 6 & 0.348 & 0.334 & 0.318 \\
\hline
\end{tabular}

Note: in the [,], before and after the comma are, respectively, the minimum and maximum value of the objective function. 
TABLE 6: Thermal power output arrangement in different scenarios $(M W \cdot h)$.

\begin{tabular}{|c|c|c|c|c|c|c|}
\hline Unit & Scenario 1 & Scenario 2 & Scenario 3 & Scenario 4 & Scenario 5 & Scenario 6 \\
\hline $\mathrm{T} 1$ & 5378 & 5404 & 5430 & 5457 & 5483 & 5512 \\
\hline $\mathrm{T} 2$ & 5047 & 5125 & 5204 & 5285 & 5367 & 5470 \\
\hline T3 & 8249 & 8344 & 8440 & 8507 & 8547 & 8565 \\
\hline $\mathrm{T} 4$ & 7755 & 7857 & 7961 & 8065 & 8172 & 8279 \\
\hline T5 & 4689 & 4706 & 4708 & 4725 & 4757 & 4774 \\
\hline T6 & 3189 & 3298 & 3411 & 3528 & 3649 & 3774 \\
\hline H1 & 9088 & 8945 & 8804 & 8665 & 8529 & 8394 \\
\hline $\mathrm{H} 2$ & 19203 & 19025 & 18848 & 18673 & 18500 & 18328 \\
\hline H3 & 3633 & 3528 & 3426 & 3326 & 3230 & 3136 \\
\hline
\end{tabular}

TABLE 7: System scheduling results compared in different scenarios.

\begin{tabular}{lcccccccccc}
\hline \multirow{2}{*}{ Scenario } & \multicolumn{3}{c}{ Fuzzy VaR and CVaR value } & \multicolumn{3}{c}{ Output allocation/MW·h } & \multirow{2}{*}{$F_{1} /$ ton } & \multirow{2}{*}{$F_{2} / 10^{5} \mathrm{~kg}$} & \multirow{2}{*}{$F_{3} / 10^{5} \mathrm{~m}^{3}$} \\
& $\mathrm{VaR}_{1}$ & $\mathrm{VaR}_{2}$ & $\mathrm{VaR}_{3}$ & $\mathrm{VaR}$ & $\mathrm{CVaR}$ & Thermal & Hydro & 31925 & 8239 & 10538 \\
Scenario 1 & 0.33 & 0.308 & 0.292 & 0.31 & 0.330 & 34307 & 31498 & 6508 & 8334 \\
Scenario 2 & 0.347 & 0.322 & 0.304 & 0.324 & 0.337 & 34734 & 31078 & 5569 & 7239 & 469 \\
Scenario 3 & 0.364 & 0.336 & 0.316 & 0.339 & 0.344 & 35154 & 30665 & 7845 & 7845 \\
Scenario 4 & 0.341 & 0.351 & 0.328 & 0.339 & 0.351 & 35567 & 30258 & 6428 & 6852 \\
Scenario 5 & 0.352 & 0.332 & 0.330 & 0.338 & 0.362 & 35974 & 2985 & 4517 \\
Scenario 6 & 0.371 & 0.354 & 0.297 & 0.342 & 0.385 & 36374 & 2985 & 5432 & 7024 & 4174 \\
\hline
\end{tabular}

different, the higher the confidence level, the bigger the weight. This phenomenon means the entropy method is rational because it could allocate weights that can reflect decision maker's risk attitude.

Use parameters and coefficients in Table 5 to build fuzzy multiobjective CVaR model, and do simulation for 6 scenarios. Table 6 lists the simulation result and output allocation in different scenarios.

Compare scenario 1, scenario 2, and scenario 3 in Table 6 and find that as the confidence level increases thermal units' output would increase, and hydropower output would decrease. That means sacrifice some thermal power consumption to increase the first-level hydropower station's storage capacity as backup service for load fluctuations. Table 7 lists scheduling operation results in different scenarios.

Compare scenario 1, scenario 2, and scenario 3 in Table 7 and find that when all objective functions' confidence levels are the same, the upgrade of confidence level requirement would promote the scheduling result close to the optimum result. However, VaR and CVaR value would also increase, in which the risk is increasing. Therefore, if a decision maker pursues better scheduling results, he must have the capacity to undertake bigger risk. Compare scenario 4, scenario 5, and scenario 6 and find when the confidence levels are different, the higher the confidence level the better the objective function value, and the higher the risk level. Those two conclusions are consistent with each other.

Then, longitudinal compared scenarios with the same or different confidence levels, it can be seen, if confidence levels of objective functions are different, system risk level would increase, but the optimization result would be better, for example, scenario 1 and scenario 4 . Therefore if the decision maker wants to optimize a specific objective, the system needs to pay more effort to achieve the goal while meeting other objective functions' requirements and system's operation risk would be increased. In uncertain load hydrothermal joint scheduling problems, better scheduling scheme means bigger risk, and smaller risk generally corresponds with worse scheduling scheme. Decision makers should balance effectiveness with their actual situation and risk tolerance to choose rational confidence level and get an accessible optimum scheduling scheme.

\section{Conclusions}

To analyse the risk that load demand uncertainty brings to hydrothermal power system joint scheduling, this paper regarded actual load and forecast error as random variable and built a multiobjective CVaR hydrothermal joint scheduling optimization model considering load demand uncertainty. According to the simulation results, the following conclusions can be concluded:

(1) Multiobjective CVaR method could effectively reflect the risk that uncertain factors bring to hydrothermal scheduling and get the optimum scheduling scheme under different risk attitudes.

(2) Fuzzy satisfaction degree theory and entropy theory could simplify CVaR model and transfer multiobjective nonlinear problems into single objective linear optimization problems, which is conducive to get the optimum scheduling scheme.

(3) Decision maker's risk attitude would directly influence system scheduling optimization scheme. The stronger the system risk tolerance, the less the power 
generation energy consumption, the less the pollutant emission and the more the storage capacity of the first-level hydropower station.

(4) This paper assumed load forecast error obeys normal distribution. But this assumption is not always real in the actual production situations. Therefore the proposed model could be improved by using load forecast error's actual distribution function.

\section{Conflict of Interests}

The authors declare that there is no conflict of interests regarding the publication of this paper.

\section{Acknowledgment}

This paper is supported by the National Science Foundation of China (Grant no. 71273090) Project Supported by the Fundamental Research Funds for the Central Universities of China (Grant no. 2015XS29).

\section{References}

[1] J. Chuanwen and E. Bompard, "A self-adaptive chaotic particle swarm algorithm for short term hydroelectric system scheduling in deregulated environment," Energy Conversion and Management, vol. 46, no. 17, pp. 2689-2696, 2005.

[2] Z.-F. Tan, G.-J. Chen, J.-B. Zhao, J.-C. Hou, and H.-Y. Jiang, "Optimization model for designing peak-valley time-of-use power price of generation side and sale side at the direction of energy conservation dispatch," Proceedings of the Chinese Society of Electrical Engineering, vol. 29, no. 1, pp. 55-62, 2009 (Chinese).

[3] Z. Hu, "Study on the pattern of China low-carbon economy," Energy of China, vol. 31, no. 11, pp. 16-19, 2009 (Chinese).

[4] X. She, A. Q. Huang, F. Wang, and R. Burgos, "Wind energy system with integrated functions of active power transfer, reactive power compensation, and voltage conversion," IEEE Transactions on Industrial Electronics, vol. 60, no. 10, pp. 45124524, 2013.

[5] L.-F. Mao, Y.-C. Jiang, J.-G. Yao, R.-H. Long, N. Li, and H. Huang, "Medium and long term load forecasting based on orthogonal signal correction and partial least-squares regression," Proceedings of the Chinese Society of Electrical Engineering, vol. 29, no. 16, pp. 82-88, 2009.

[6] J. Qin S, "Partial least squares regression for recursive system identification," in Proceedings of the 32nd IEEE Conference on Decision and Control, vol. 3, pp. 2617-2622, IEEE, San Antonio, Tex, USA, December 1993.

[7] D. Niu, S. Cao, and J. Lu, Power Load Forecasting Technology and Its Application, China Electric Power Press, Beijing, China, 2009, (Chinese).

[8] X. Chen, C. Kang, and M. Chen, "Short term probabilistic forecasting of the magnitude and timing of extreme load," Proceedings of the CSEE, vol. 31, no. 22, pp. 64-72, 2011 (Chinese).

[9] K. Jisong and L. Minqiang, "Clusterimulti-Small area forecasting and spatial resolution identification in spatial load forecast," Journal of Systems Engineering, vol. 4, no. 2, pp. 42-53, 1989 (Chinese).
[10] Z.-F. Tan and J.-L. Zhang, "Day-ahead electricity price forecasting based on multi-factor wavelet analysis and multivariate time series models," Proceedings of the Chinese Society of Electrical Engineering, vol. 30, no. 1, pp. 103-110, 2010.

[11] Y. Feng, R. Li, A. Sudjianto, and Y. Zhang, "Robust neural network with applications to credit portfolio data analysis," Statistics and Its Interface, vol. 3, no. 4, pp. 437-444, 2010.

[12] N. Lu, J.-Z. Zhou, and Y.-Y. He, "Particle swarm optimizationbased neural network model for short-term load forecasting," Power System Protection and Control, vol. 38, no. 12, pp. 65-68, 2010 (Chinese).

[13] X. Wu, X. Wang, J. Li, J. Guo, K. Zhang, and J. Chen, "A joint operation model and solution for hybrid wind energy storage systems," Proceedings of the Chinese Society of Electrical Engineering, vol. 33, no. 13, pp. 10-17, 2013.

[14] Z. Hu, H. Ding, and T. Kong, "A joint daily operational optimization model for wind power and pumped-storage plant," Automation of Electric Power Systems, vol. 36, no. 2, pp. 36-57, 2012.

[15] Z.-G. Hu, X.-D. Tan, B.-G. Shan, C.-B. Li, and M.-J. Xu, "Application of intelligent engineering and agent to DSM compensation mechanism modelling," Proceedings of the Chinese Society of Electrical Engineering, vol. 28, no. 13, pp. 125-131, 2008 (Chinese).

[16] J. H. Grake, "Optimum operation of a hydrothermal system," IEEE Transactions on Power Apparatus and Systems, vol. 80, no. 3, pp. 242-250, 1962.

[17] W. W.-G. Yeh, L. Becker, S. Hua, D. Wen, and J. Liu, "Optimization of real-time hydrothermal system operation," Journal of Water Resources Planning and Management, vol. 118, no. 6, pp. 636-653, 1992.

[18] Y. Qian, H. Zhang, D. Peng, and F. Xia, "Orbit purification of generator unit based on a new generalized particle swarm optimization method," Proceedings of the Chinese Society of Electrical Engineering, vol. 32, no. 2, pp. 130-137, 2012.

[19] X. Wu, B. Zhang, X. Yuan, G. Li, G. Luo, and Y. Zhou, "Solutions to unit commitment problems in power systems with wind farms using advanced quantum-inspired binary PSO," Proceeding of the CSEE, vol. 33, no. 4, pp. 45-52, 2013.

[20] S. Lou, X. Yu, X. Xiong et al., "Unit commitment using improved discrete particle swarm optimization algorithm," Proceedings of the CSEE, vol. 25, no. 8, pp. 30-35, 2005 (Chinese).

[21] L. V. L. Abreu, M. E. Khodayar, M. Shahidehpour, and L. $\mathrm{Wu}$, "Risk-constrained coordination of cascaded hydro units with variable wind power generation," IEEE Transactions on Sustainable Energy, vol. 3, no. 3, pp. 359-368, 2012.

[22] W. Duan, Z. Hu, S. Wu, Y. Zhou, and M. Yao, "Dynamic simulation of economic policy and electricity demand by agents response equilibrium model," Proceedings of the Chinese Society of Electrical Engineering, vol. 34, no. 7, pp. 1206-1212, 2014 (Chinese).

[23] M. Jiang, B. Z. Jiang, Z. Q. Meng, and X. Yu, "Risk measure and strategy of portfolio investment based on the multiobjective CVaR model," Mathematics in Economics, vol. 24, no. 4, pp. 385391, 2007.

[24] H.-Y. Wu, X.-H. Guan, Q.-Z. Zhai, and F. Gao, "Short-term hydrothermal scheduling using mixed-integer linear programming," Proceedings of the Chinese Society of Electrical Engineering, vol. 29, no. 28, pp. 82-88, 2009. 
[25] J. Wu and L. Tang, "Stochastic optimization scheduling method for hydrothermal power systems with stochastic loads," Proceedings of the Chinese Society of Electrical Engineering, vol. 32, no. 28, pp. 36-43, 2012.

[26] M. Qi, Z. Fu, Y. Jing, and Y. Ma, "A comprehensive evaluation method of power plant units based on information entropy and principal component analysis," Proceedings of the Chinese Society of Electrical Engineering, vol. 33, no. 2, pp. 58-65, 2013. 


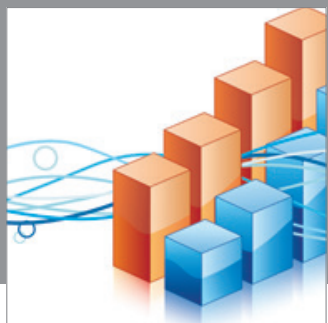

Advances in

Operations Research

mansans

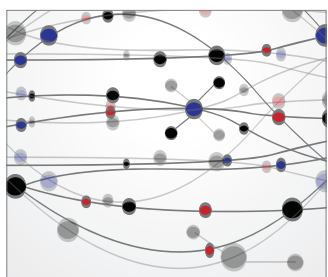

The Scientific World Journal
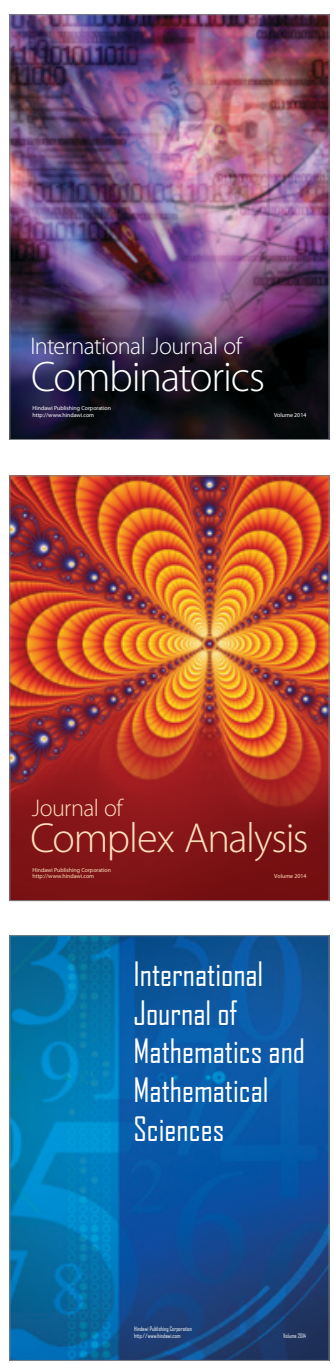
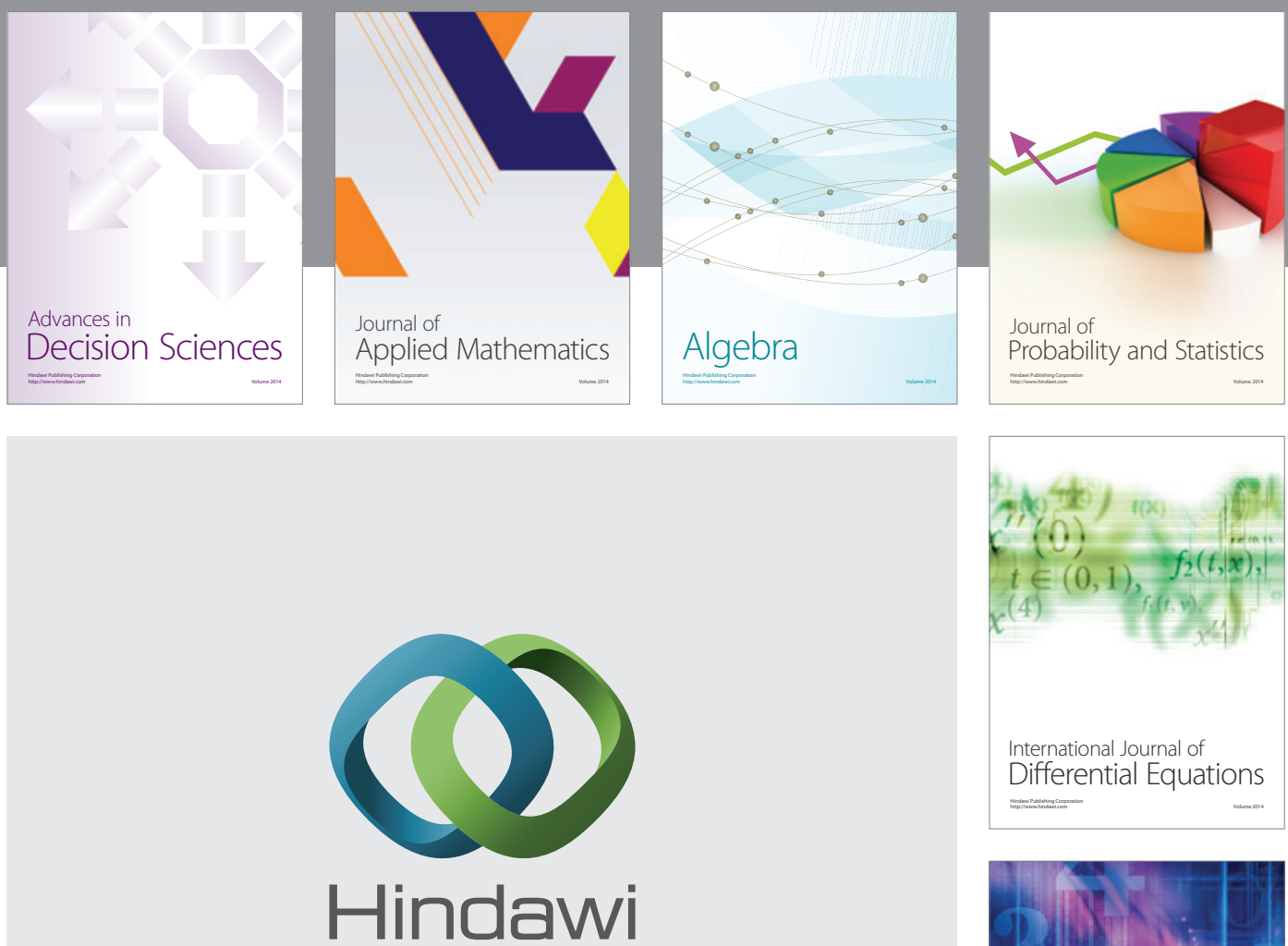

Submit your manuscripts at http://www.hindawi.com
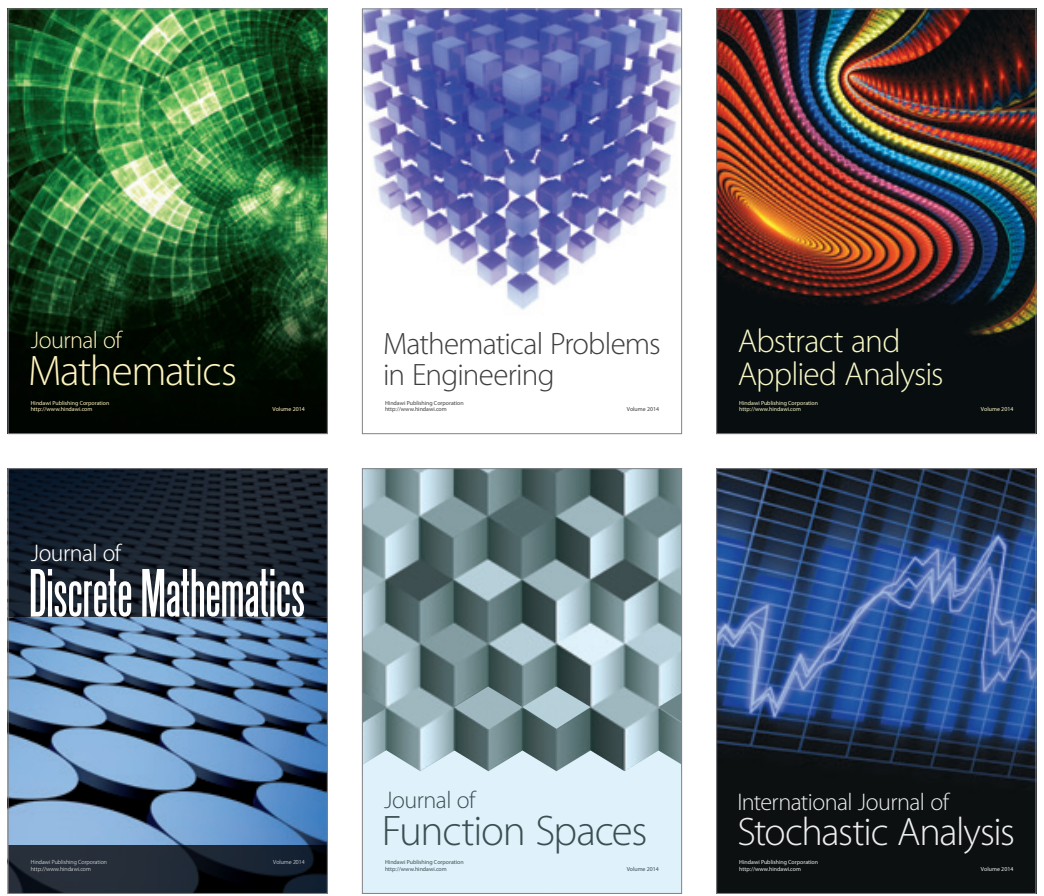

Journal of

Function Spaces

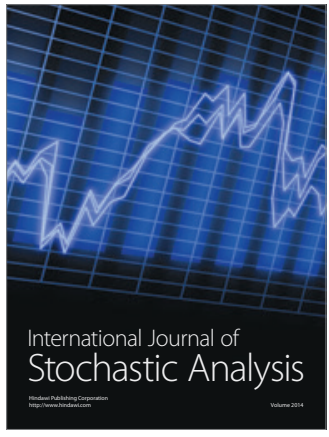

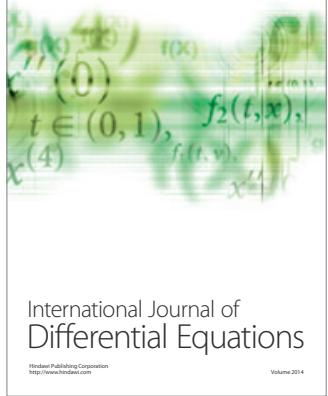
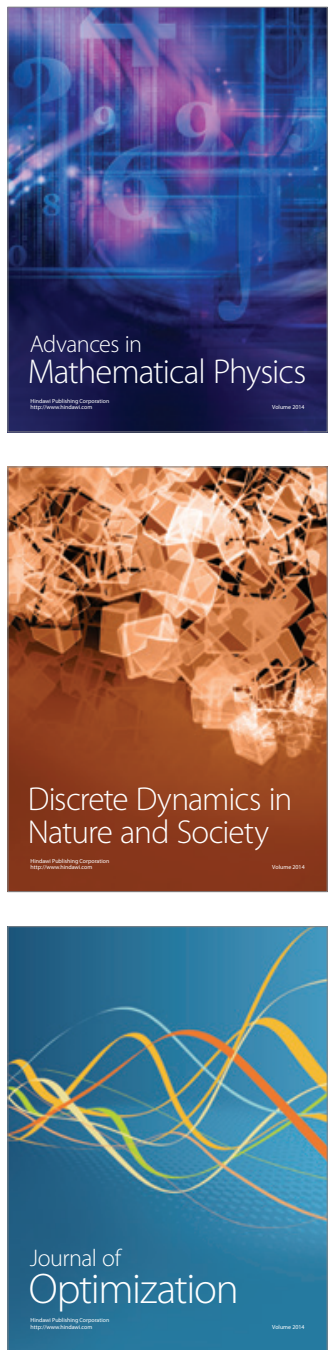